\title{
Les automatismes dans l'activité : pour une réhabilitation et un bon usage
}

Automatisms into activity: for a restoration and a good use

Los automatismos en la actividad: en pos de una reivindicación y de un buen uso de los mismos

Jacques Leplat

\section{OpenEdition}

\section{Journals}

Édition électronique

URL : http://journals.openedition.org/activites/1797

DOI : 10.4000/activites. 1797

ISSN : $1765-2723$

\section{Éditeur}

ARPACT - Association Recherches et Pratiques sur les ACTivités

Référence électronique

Jacques Leplat, «Les automatismes dans l'activité : pour une réhabilitation et un bon usage », Activités [En ligne], 2-2 I octobre 2005, mis en ligne le 01 octobre 2005, consulté le 30 avril 2019. URL http://journals.openedition.org/activites/1797 ; DOI : 10.4000/activites.1797

\section{(c) (i) $9 \Theta$}

Activités est mis à disposition selon les termes de la licence Creative Commons Attribution - Pas d'Utilisation Commerciale - Pas de Modification 4.0 International. 


\title{
Les automatismes dans l'activité : pour une réhabilitation et un bon usage
}

\author{
Jacques Leplat \\ Directeur honoraire à l'École Pratique des Hautes Études \\ 41, rue Gay-Lussac, 75005 Paris
}

\begin{abstract}
Automatisms into activity: for a restoration and a good use

Automatisms have a large place in the activity, but often they are put forward for their negative consequences, in particular for the role they play in the production of errors. Revisiting the nature of the automatisms, the present text shows they have also properties which can have a positive role into activity. To proceed to their rehabilitation, general features of automatims will be recalled, then characteristics and conditions of their development. A classification of the automatisms will be proposed. For a better understanding of the place of automatisms into activity, we will examine their place in different models of activity commonly used in ergonomics. At least, some ways will be suggested for a relevant use of automatisms which takes advantage of their qualities and reduces their negative effects.
\end{abstract}

\section{KEYWORDS}

Activity, attention, automatism, work load, coupling, training, habitus, scheme, routine.

\begin{abstract}
«Car il ne faut pas se méconnaître : nous sommes automate autant qu'esprit; et de là vient que l'instrument par lequel la persuasion se fait n'est pas la seule démonstration. Combien y a-t-il peu de choses démontrées ! Les preuves ne convainquent que l'esprit. La coutume fait nos preuves les plus fortes et les plus crues; elle incline l'automate qui entraîne l'esprit sans qu'il y pense »

(Pascal, Pensées, p. 1119-20)
\end{abstract}

Le mot d'automatisme revient souvent dans les textes d'ergonomie et de psychologie du travail, notamment dans ceux concernant l'analyse de l'activité. Mais la fréquence de cette référence n'est pas un signe d'estime ! L'automatisme est marqué d'un certain dédain : il a tendance à être considéré comme une forme d'activité inférieure, machinale, dépourvue d'intelligence. Il est même parfois vu comme un trait d'animalité chez l'homme. C'est surtout pour relever leurs conséquences négatives qu'il est fait référence aux automatismes. Ces derniers sont dénoncés comme sources d'erreurs, de sclérose, d'obstacles à l'acquisition. Ces inconvénients sont bien réels, mais ils ne doivent pas conduire à ne considérer les automatismes que sous leur angle négatif : leurs défauts sont souvent l'envers de leurs qualités. Le présent texte voudrait contribuer à la réhabilitation des automatismes en montrant qu'on peut en faire un bon usage qui permette de profiter de leurs avantages en réduisant leurs éventuels inconvénients.

Cet article a été conçu dans une perspective ergonomique et voudrait suggérer qu'une meilleure connaissance des caractéristiques des automatismes peut enrichir l'analyse de l'activité comme la recherche des solutions aux problèmes que cette analyse fait apparaitre. Il ne traitera pas des multiples facettes des automatismes, mais il voudrait en faire ressortir quelques unes propres à nourrir la réflexion et être exploitées dans les pratiques. A cette fin, on rappellera d'abord quelques traits géné- 
raux des automatismes avec un petit rappel historique sur les situations qui ont contribué à l'émergence de la notion. On commentera ensuite quelques caractéristiques qui marquent le développement des automatismes et on esquissera une classification possible des automatismes. Une partie examinera quelle place est faite aux automatismes dans quelques théories de l'activité auxquelles il est fait référence en ergonomie. Enfin, une dernière partie traitera d'une manière plus concrète des conditions et des avantages d'un bon usage des automatismes en ergonomie. Cet article qui rappelle beaucoup de connaissances classiques a une visée pédagogique et voudrait contribuer au perfectionnement des outils de ceux qui s'intéressent à l'analyse de l'activité.

\section{1.- Traits généraux des automatismes}

Automatisme, processus automatisé, activité automatisée sont des termes souvent employés comme synonymes, mais qui n'évitent pas des ambiguïtés. «Automatisme » est un mot qui a un sens précis en ingénierie mécanique, mais qui s'ajuste mal au domaine de l'activité. "Activité automatisée » n'est pas, non plus, sans inconvénient, car la notion d'activité a une signification précise dans certaines théories, qui ne s'accommode pas de la notion d'automatisme. Il en est ainsi, comme on le verra par la suite, pour la théorie russe de l'activité dans laquelle l'automatisme est lié à la notion d'opération, celle-ci n'étant qu'une composante de l'action, elle-même composante de l'activité. Dans ce cas, il faudrait parler d'automatisme dans l'activité ou de composante automatisée de l'activité ou, encore, d'opération automatisée. Comme nous ne nous situerons pas dans le cadre d'une théorie particulière, nous garderons en général le terme d'automatisme, laissant aux auteurs cités le choix de leur terminologie.

Pour mieux cerner cette notion d'automatisme, on a interrogé deux vocabulaires. Celui de Piéron est assez pauvre sur le mot d'automatisme : son champ de référence est essentiellement psychophysiologique ou psychopathologique. Finalement, il distingue « des automatismes primaires, innés, et des automatismes secondaires, acquis » : ce sont ces derniers qui nous intéresseront ici.

Le Vocabulaire de sciences cognitives de Houdé, Kayser, Koenig, Proust et Rastier (1998) est plus loquace et comporte deux divisions, Psychologie et Neuroscience. La première, dirigée par Lautrey, est introduite par le paragraphe suivant : «En psychologie cognitive, le traitement automatique de l'information est généralement caractérisé par l'absence de coût attentionnel, l'absence de contrôle, l'absence de conscience, le parallélisme des opérations et la rapidité d'exécution.» La suite reprend ces caractéristiques en les détaillant. On retiendra aussi le premier paragraphe du texte d'automatisme pour la division «Neuroscience» (rédigée par Sieroff) : «dans les années 1880, John Hughlings Jackson, médecin anglais, insistait sur la dissociation automatico-volontaire dans le comportement. Il avançait également une hypothèse des mécanismes cérébraux des processus automatiques. Pour lui, les automatismes faisaient appel à des processus mieux organisés dans le système nerveux et donc moins fragiles devant une lésion partielle de celui-ci. Au contraire, les processus les plus volontaires, plus récents sur le plan phylogénétique, étaient considérés comme plus complexes et moins bien organisés, moins bien implantés dans le système nerveux et donc plus fragiles. Il peut donc apparaître que les automatismes "appris", c'est-à-dire les automatismes cognitifs, correspondent à des réseaux largement ancrés dans le système nerveux et que seuls les processus attentionnels correspondraient à un ensemble d'aires anatomiquement et fonctionnellement distinctes. (...) En fait, la question du soubassement neuronal des automatismes cognitifs est certainement l'une des questions les moins bien résolues en neuroscience. » Ce dernier texte est mentionné pour rappeler que les processus automatiques ont des supports cérébraux qui sont aussi un objet de recherche important. Cette facette des automatismes ne sera pas abordée ici où nous nous limiterons aux aspects psychologiques. 
Les précédentes définitions auront déjà fait ressortir des propriétés des automatismes. Ces propriétés ont souvent été utilisées pour diagnostiquer le caractère automatique d'une activité. Mais, comme le remarque Logan (1988), «le diagnostic de l'automaticité est plein de problèmes. Les différents chercheurs utilisent différentes listes de propriétés et sont en désaccord sur le caractère nécessaire et suffisant de ces propriétés » (p. 515). La difficulté fondamentale tient au fait que le caractère automatique est lié à des mécanismes internes qui ne sont pas directement observables, mais doivent être inférés. Nous retiendrons quelques unes de ces propriétés en nous inspirant, notamment, des textes de Perruchet (1988) et de Logan (1988), et en commençant par les deux plus importantes. Nous essayerons de les décrire en référence à des situations de travail.

\section{1.- « L'absence de charge mentale »}

C'est, selon Perruchet, l'une des deux propriétés principales des automatismes, celle qui semble faire l'objet d'un large accord. Ravaisson (1837) notait déjà que " par l'exercice répété ou prolongé, nous apprenons à proportionner la quantité d'effort et à en choisir le point d'application conformément à la fin que nous voulons atteindre; et en même temps s'efface la conscience de l'effort » (p. 35). La notion de charge mentale fréquemment utilisée en psychologie du travail et en ergonomie n'est pas une notion claire et elle a fait l'objet de nombreuses critiques dont on trouvera quelquesunes dans Jourdan et Theureau (2002). C'est à son imprécision qu'elle doit, sans doute, ses nombreux synonymes : effort mental, coût cognitif, charge attentionnelle, astreinte mentale, ressources mentales (Tricot \& Chanquoy, 1996).

Pour les situations qui nous concernent le plus directement ici, on pourra retenir, des travaux conduits autour de la charge mentale, ceux qui ont fait appel à la méthode de la double tâche. S'il fallait les caractériser sommairement, on pourrait dire qu'ils reposent sur deux hypothèses. D'une part que les possibilités d'exécution d'une tâche sont limitées, d'autre part, qu'une manière d'évaluer la charge représentée par une exécution particulière d'une tâche peut être d'estimer la fraction de ces possibilités globales qu'elle requiert. Il existe de nombreux travaux expérimentaux conduits autour de ce schéma qui a naturellement reçu des opérationnalisations précises (Leplat, 2002). Des méthodes dites de la double tâche ont mis en œuvre ces hypothèses. Comme elles sont bien connues, on donnera simplement le principe de l'une d'entre elles. Il consiste à adjoindre à la tâche primaire dont on veut évaluer la charge qu'elle représente pour l'activité, une tâche secondaire que l'opérateur doit effectuer en parallèle avec la première sans nuire à l'efficacité de l'exécution de celle-ci. L'efficacité de l'exécution de la tâche secondaire choisie comme aisément mesurable sera d'autant plus grande que la tâche primaire représentera une charge plus faible. Ce procédé correspond à une certaine intuition de la notion de charge. Par exemple, si l'on veut évaluer la charge représentée par la conduite automobile avec ce type de méthode, on pourra choisir comme tâche secondaire des opérations à exécuter (additions, reconnaître des suites particulières de sons, etc.). On conçoit qu'à mesure que le conducteur devient plus expérimenté, c'est-à-dire a automatisé certains aspects de la conduite, il devient aussi plus capable d'améliorer sa performance à la seconde tâche. Ainsi, on dira que le caractère automatique d'une action peut s'exprimer par le fait qu'elle est exécutable en parallèle avec une autre tâche, ou que son exécution est compatible avec celle d'autres tâches. Il n'est pas question ici de rapporter les critiques qui sont adressées à ce genre de méthode qui a été largement exploitée pour évaluer le degré d'automaticité d'une opération ou même d'une exécution d'une tâche plus complexe.

\section{2.- « L'absence de contrôle attentionnel »}

C'est la seconde des deux propriétés principales attribuées par Perruchet aux automatismes. «On utilise de façon équivalente, pour désigner ce second critère, les termes d'obligatoire, non optionnel, non délibéré ou autonome » (p. 33). L'auteur voit comme justification à cette propriété « la nécessité 
de déployer une activité inhibitrice pour mettre un terme au déroulement d'un processus ... » (id.). Wallon (1938) a exprimé ainsi cette caractéristique pour les automatismes manuels : « Mais l'automatisme n'a de sûreté que dans la mesure où il se dérobe à l'attention du sujet et où sa participation reste comme anonyme dans l'acte en train de s'exécuter ; par exemple, le mouvement des doigts sur les touches, quand le pianiste a les yeux sur sa partition. Que brusquement, au contraire, la fantaisie vienne au marcheur ou au pianiste d'imaginer le contact de ses pieds avec les marches de l'escalier ou de ses doigts avec les touches, et l'automatisme a bien des chances d'accrocher » (p. 236).

L'absence de contrôle attentionnel apparaît nettement dans les situations où l'automatisme continue à se dérouler alors que ses conditions de validation ne sont plus remplies. Cette caractéristique se révèle dans la production de certains types d'erreur dits «ratés » («slips ») et lapsus qu'ont bien décrits les analystes (Norman, 1981, Leplat, 1985, Reason, 1990). Ce sont les erreurs qui correspondent au cas où le sujet a fixé un but correct à son action, mais échoue dans la réalisation de ce but, laissant capturer l'action planifiée par un automatisme. C'est le cas du voyageur qui a l'habitude d'emprunter un certain trajet pour aller quotidiennement à son travail, et qui, un jour, devant se rendre à une réunion dans un autre lieu se retrouve sur le lieu de son travail, comme si l'automatisme avait capté l'action projetée du conducteur (c'est notamment le cas quand le début du parcours est commun aux deux destinations).

Une situation souvent évoquée aussi pour témoigner de l'absence de contrôle attentionnel est celle dite de l'effet Stroop qui apparait lorsqu'on demande à un sujet de nommer la couleur d'un mot imprimé et que ce mot désigne une couleur différente (exemple, le mot « jaune » est écrit en bleu). Le temps de dénomination augmente dans ce cas d'interférence (entre le mot et la couleur) et il peut même se produire des erreurs. Les résultats obtenus dans des situations de même type conduisent à penser que l'automatisme mis en jeu par la lecture du mot se déclenche de manière non intentionnelle et interfère avec l'objectif de dénomination de la couleur.

\section{3.- Autres propriétés}

D'autres propriétés sont attribuées aux automatismes, qui sont souvent en rapport direct avec les précédentes, plus fondamentales. Perruchet (1988) attribue une place particulière à l'inconscience « opérationnalisée par l'incapacité des sujets à verbaliser, ou plus généralement à témoigner intentionnellement par une réponse symbolique de la nature d'un processus ou d'un événement » (p. 35). Wallon (1942) avait déjà noté qu' "il arrive que la conscience n'ait plus part à des processus dont les termes avaient une valeur représentative mais l'ont perdue. C'est le cas des mouvements devenus automatiques. » Nous n'insisterons pas sur cette propriété qui sera abordée quand il sera question des compétences.

Diverses propriétés sont aussi évoquées, assez directement liées à l'absence de charge mentale : la principale est sans doute la résistance aux facteurs perturbateurs comme les contraintes de vitesse et les tâches interférentes. Cette propriété a été exploitée pour tester le degré d'automatisme présenté par une tâche : on évalue ce degré à partir de la sensibilité de l'activité étudiée aux contraintes de vitesse ou aux tâches perturbatrices qu'on peut lui adjoindre. Les vieilles études ergonomiques sur les stéréotypes et la compatibilité en fourniraient de nombreux exemples (par exemple, Leplat, 1972, p. 96 sq.).

Shiffrin et Dumais (1980) ont signalé aussi deux autres propriétés. La continuation est cette propriété selon laquelle les processus automatiques ont tendance à se dérouler jusqu'à leur terme (« completion») à moins qu'un effort soit fait pour les interrompre, ou qu'un autre processus automatique utilise les mêmes ressources. Quand l'automatisme est interrompu, il peut difficilement être poursuivi et requiert d'être repris au début pour être achevé. Par exemple, si quelqu'un est interrompu dans l'énoncé de mémoire d'un poème antérieurement appris, il est souvent obligé de reprendre au début pour réciter tout le poème. 
Cette propriété est à rapprocher de la propriété dite d'autonomie définie ainsi par Logan (1988) : « un processus est autonome s'il peut commencer et se poursuivre jusqu'à son achèvement sans intention », ce qui ne signifie pas, ajoute l'auteur, qu' «il ne puisse être intentionnellement mis en œuvre et guidé vers son achèvement » (p. 513). De même, comme le note le même auteur, l'autonomie n'entraîne pas que le processus automatique ne soit pas étroitement contrôlé, ce dont on peut voir une preuve dans le fait que les automatismes peuvent être rapidement inhibés lorsque survient une erreur dans leur déroulement (p. 512). Wallon (1942) avait donné un bel exemple de ce phénomène avec l'activité de la tricoteuse experte qui a automatisé son activité, mais qui, «s'il lui arrive par accident de laisser tomber une maille, instantanément ses yeux se portent sur son ouvrage... »; elle doit alors, comme le dit Wallon agir un peu comme l'apprentie en mettant en jeu un «effort d'attention par un acte de conscience. »

Cette caractéristique est parente de celle dite par les mêmes auteurs d'indivisibilité, selon laquelle «il peut être difficile de commencer en son milieu l'exécution d'une séquence automatisée » (p.122), par exemple en commençant à chanter une chanson bien connue au milieu d'une phrase. On peut lier à cette dernière propriété la difficulté de modifier un processus automatisé en en retranchant certaines parties ou en les remplaçant par d'autres. Il faut alors recourir à un nouvel apprentissage prolongé faute duquel l'automatisme primitif a tendance à réapparaître.

On a signalé aussi comme propriété la dépendance réduite à l'égard de l'environnement. Qu'il s'agisse du déclenchement ou du contrôle de l'activité, l'automatisme ne prend en compte qu'un modèle souvent sommaire de l'ensemble des conditions dans laquelle se déroule l'exécution de la tâche. Il n'en retient que les traits suffisants dans les circonstances habituelles. Cette ignorance des autres traits est une source d'économie, mais elle peut aussi se révéler fâcheuse quand l'absence ou la modification d'un de ces traits négligés invalide l'action en cours. Par exemple, un opérateur met en marche la machine sur laquelle il travaille habituellement sans s'apercevoir qu'un objet bloque son fonctionnement : d'où des dégâts. De nombreux accidents relèvent d'un tel mécanisme. Cette cécité relative à l'égard de l'environnement est une des sources du manque d'adaptabilité imputé aux automatismes. Cependant, l'énoncé de cette propriété a un caractère ambigu, car on a pu dire aussi que les automatismes étaient très liés à l'environnement au sens où, quand ils s'exercent dans des conditions très stables, ils conduisent à prendre pour critiques des traits de la tâche ou de l'environnement (parce qu'ils sont généralement associés aux traits véritablement critiques) qui ne sont pas pertinents à son exécution : par exemple, un opérateur va utiliser un produit à la place d'un autre parce que leur place habituelle a été intervertie.

\section{4.- Rappel historique}

On s'est intéressé très tôt aux phénomènes désignés maintenant sous le nom d'automatisme, et il est instructif d'examiner ce qu'il ressort de ces travaux qui ne sont pas sans éclairer encore nos analyses actuelles. C'est autour de la notion d'habitude que se sont organisés ces travaux anciens dont on ne donnera ici qu'un bref aperçu. Ravaisson (1837) est le premier auteur qui vient à l'esprit avec son livre intitulé justement «De l'habitude ». Dans la présentation que fait Towarniki de la réédition de 1997, celui-ci écrit : "l'habitude qu'analyse Ravaisson est celle que nous vivons tous, celle que nous connaissons et qui fait partie de notre être. L'être de l'homme comporte une dimension d'habitude. On ne vit pas sans habitude. L'être humain prend des habitudes, se choisit des habitudes, se structure par l'habitude : il y gagne et d'un autre côté, il y perd, il y a risque d'engourdissement dans la routine » (p. 11-12). Le texte de Ravaisson a de quoi surprendre le psychologue de notre temps par son langage et par le contexte philosophique dans lequel il s'inscrit, mais il révèle des traits essentiels de ce que nous appelons aujourd'hui automatisme. Ainsi déclare-t-il que « Non seulement, donc, les mouvements que l'habitude soustrait graduellement à la volonté ne sortent pas par cela même de la sphère de l'intelligence pour passer sous l'empire d'un mécanisme aveugle ; mais 
ils ne sortent pas de la même activité intelligente où ils avaient pris naissance » (p. 80). Cette idée que l'habitude ne sort pas de la sphère de l'intelligence évoque ce texte de Merleau-Ponty (1945) : « Faut-il mettre à l'origine de l'habitude un acte d'entendement qui en organiserait les éléments pour s'en retirer ensuite ? (en note : comme le pense par exemple Bergson quand il définit l'habitude comme "le résidu fossilisé d'une activité spirituelle") » (p. 167). Certes, Ravaisson n'en tirera pas les conséquences de Merleau-Ponty, mais sa remarque que l'habitude n'est pas déconnectée de tout contrôle de l'activité est importante et sera examinée dans la partie 3. Ravaisson apporte aussi des idées originales qui seront reprises au siècle suivant. Il note ainsi qu'avec l'habitude « l'intervalle que l'entendement se représentait entre le mouvement et son but diminue donc peu à peu. (...). À la réflexion qui parcourt et qui mesure les distances des contraires, les milieux des oppositions, une intelligence immédiate succède par degrés, ou rien ne sépare l'objet de la pensée » (p. 78). On trouve là des idées propres à éclairer la réflexion sur les automatismes. $\mathrm{Si}$, comme le souligne Ravaisson, les habitudes s'acquièrent «par une suite de degrés imperceptibles » (p. 79), elles ne se distinguent plus d'une conception élargie des automatismes qui n'identifient pas ceux-ci à une forme extrême et complètement stéréotypée de l'action.

La notion d'automatisme est très associée au nom de Pierre Janet dont la thèse, «L'automatisme psychologique » présentée en 1889 a fait l'objet de nombreuses rééditions. Pour cet auteur (Janet, 1973), l'automatisme psychologique est une «activité qui tend à conserver et à répéter » (p. 12) : il l'oppose à «l'activité qui synthétise, qui organise les phénomènes du présent » (id.). Ces activités « dépendent l'une de l'autre, mais elles se limitent et se règlent réciproquement et ce n'est que la diminution de l'activité de synthèse actuelle, affaiblissement marqué par toutes sortes de symptômes, qui permet le développement exagéré de l'automatisme ancien» (p. 12). La perspective de recherche de Janet est psychopathologique voire médicale, «car l'automatisme ne se manifeste d'une manière aussi nette et aussi exagérée que dans les états pathologiques » (p. 19). Il y a sans doute là une voie de recherche intéressante à lier à celle de l'étude des supports neurologiques des automatismes, mais qui ne sera pas abordée ici.

Un peu plus d'un siècle après Ravaisson, Guillaume (1947) allait consacrer un ouvrage à « La formation des habitudes». L'habitude y était définie "par opposition avec l'instinct, comme une manière d'être - d'agir, de sentir ou de penser - acquise, faut-il ajouter que cette acquisition est due à la répétition des actions subies ou faites par l'être qui acquiert cette habitude » (p. 18). Guillaume souligne à ce propos le caractère équivoque de la notion de répétition. «Il y a contradiction entre l'idée de répétition, au sens rigoureux de répétition du même acte, et l'idée d'acquisition d'une façon d'agir nouvelle. Si on répétait toujours le même acte, il n'y aurait pas de changement, on n'apprendrait jamais rien. C'est parce qu'on ne se borne pas à reproduire qu'on apprend, qu'on progresse qu'on s'adapte » (id.). Le livre de Guillaume, puis la psychologie de l'apprentissage devaient beaucoup souligner ce point de vue. Par exemple, l'acquisition de la vitesse d'exécution qui marque souvent une propriété importante de l'automatisme résulte non pas de la reproduction à l'identique d'une action qui ne varierait que par son allure, mais cette acquisition résulte d'un réorganisation de l'action (Leplat, 1970). C'est seulement après un certain nombre de répétitions que «l'acte s'est stabilisé et la répétition semble devenir réelle. Une confusion s'établit facilement entre les répétitions réelles de l'acte appris et les pseudo-répétitions qui ont servi à l'apprendre » (Guillaume, 1947, p. 19). Ce qui reste permanent entre ces différents actes serait «l'unité de l'intention et de la signification » (id.). On pourrait avancer que l'habitude, au sens de Guillaume, tend vers l'automatisme à mesure qu'elle se stabilise.

Guillaume aborde aussi le problème des rapports entre automatismes dans les passages qu'il consacre aux interférences d'habitudes : «Les réactions des habitudes les unes sur les autres ne se manifestent pas seulement par le transfert, c'est-à-dire par des effets favorables, mais aussi par des effets défavorables, par des inhibitions » (p. 118). Il distingue deux types d'inhibitions (qu'on peut appliquer aussi aux automatismes) : l'inhibition rétroactive quand l'acquisition d'un nouvel automatisme 
perturbe ou dégrade celui déjà construit avec lequel il interfère, et l'inhibition proactive quand la présence d'un automatisme constitue un obstacle pour l'acquisition d'un nouveau. Enfin, on retiendra, de ce riche livre de Guillaume, la critique qu'il adresse à cette caractéristique que les «classiques » attribuent à l'habitude comme «tendance à faire l'acte habituel par le besoin de cet acte »(p. 20). Il note qu' «il est très douteux que toute habitude crée un besoin » (id.) et il le justifie - ceci valant aussi bien pour les automatismes - en remarquant qu'on peut savoir faire un acte sans en éprouver le besoin : « on ne souffre pas de le faire si on n'a pas de raison de le faire » (id.).

\section{2.- Développement et classification des automatismes}

Les automatismes ne se constituent pas immédiatement et leur développement dépend d'un certain nombre de conditions qu'il est utile de connaître pour mieux comprendre et maîtriser ce développement : nous en examinerons quelques-unes. Nous proposerons ensuite le principe d'une classification des automatismes à partir des caractéristiques de la tâche à l'exécution de laquelle elles correspondent. Les deux thèmes de cette partie devraient contribuer à mieux cerner les problèmes soulevés par la gestion des automatismes et leur bon usage.

\section{1.- La constitution et l'évolution des automatismes.}

On peut distinguer trois grands modes d'acquisition des automatismes dans le travail. - Un mode d'acquisition par imprégnation. C'est un mode d'acquisition non intentionnel : on apprend sans en avoir l'intention. C'est ainsi qu'ont été acquis les multiples automatismes qui jalonnent la vie quotidienne : ceux qui sont mis en jeu dans un repas, dans l'ouverture d'une porte, dans un déplacement fréquent comme celui de se rendre à son lieu de travail ou d'en revenir, etc. - Un mode d'acquisition ayant un but plus ou moins explicitement défini et centré sur une tâche particulière. Il concerne les automatismes acquis dans une situation de travail à partir de l'observation ou des conseils des collègues : il répond souvent au conseil «fais comme je fais. »C'est, dans de nombreux cas, le mode d'acquisition propre au bricoleur. On peut y rattacher aussi le mode d'acquisition par essais et erreurs. - Un mode d'acquisition explicitement contrôlé. Son exemple le plus typique est fourni par les formations scolaires et professionnelles. L'acquisition est guidée par les connaissances et les aides fournies aux opérateurs et elle s'accompagne d'un exercice systématique, nécessaire au passage à l'automatisme. Dans la présente partie seront envisagés quelques-uns des mécanismes qui ont été mis en évidence dans la constitution des automatismes.

\subsection{1.- Le rôle essentiel de la pratique}

Dans le chapitre qu'ils ont consacré à l'étude de la pratique, chapitre que nous exploiterons ici, Newell et Rosenbloom (1980) déclarent dans leur premier paragraphe : «Presque toujours, la pratique apporte une amélioration, et plus de pratique, plus d'amélioration. Nous nous attendons tous à ce que l'amélioration avec la pratique soit omniprésente, quoique, évidemment, des limites existent dans sa portée et son étendue. Considérons seulement les expériences de laboratoire : nous ne nous attendons pas à ce que les gens exécutent une tâche expérimentale correctement sans au moins quelque pratique ; et nous concevons toutes nos expériences psychologiques avec un œil sur l'influence déconcertante des effets de la pratique » (p. 1). Un peu plus loin, ils notent que «le développement d'un processus automatisé semble toujours être lié à une pratique étendue et, ainsi, les notions d'habileté (skill) et de pratique (practice) deviennent de nouveau centrales » (p. 2).

Les études classiques sur l'apprentissage montrent bien cet effet de la pratique avec généralement cette idée qu'après un certain nombre d'essais, les résultats plafonnent. Quelquefois peuvent apparaitre plusieurs plateaux successifs interprétés comme issus des changements de méthode. Or, lorsque les expériences sont poursuivies un très long temps, avec un grand nombre de répétitions, les 
performances peuvent continuer à s'améliorer selon un modèle typique. « Il existe une loi quantitative omniprésente ('ubiquitous') de la pratique : elle apparaît suivre une loi puissance, c'est-à-dire que le logarithme du temps d'exécution d'une tâche est plus ou moins linéairement lié au logarithme du nombre d'essais. Nous nous référerons à cette loi comme la loi linéaire d'apprentissage log-log ou comme la loi puissance de la pratique » (p. 2). Newell et Rosenbloom ont retrouvé cette loi pour un ensemble d'activités, élémentaires, mais aussi complexes, à dominante motrice comme cognitive, et ils en présentent des graphiques éloquents. Ils discutent aussi de l'axiomatique de quelques modèles de l'apprentissage compatibles avec cette loi puissance : ces développements techniques n'entrent pas dans le cadre de cet article, mais sont très instructifs.

L'amélioration continue de la performance avec la pratique suggère la possibilité de parler de degré d'automatisation, ce qui impliquerait que les propriétés des automatismes pourraient être plus ou moins réalisées selon la durée de cette pratique.

\subsection{2.- L'uniformité (consistency) des conditions de l'apprentissage}

Le rôle de la stabilité des conditions critiques d'une exécution à l'autre a été bien établi dans les expériences de Schneider et Shiffrin (citées plus loin). Cependant, Shiffrin et Dumais (1980) mentionnent aussi des expériences montrant que «l'automatisme peut se développer dans des conditions où l'uniformité n'est pas parfaite » (p. 126). Cette conclusion est importante en ce qui concerne les situations de travail dans lesquelles la situation d'uniformité est très relative. À un certain niveau de finesse, toutes les situations sont différentes et pourtant il existe des automatismes dans le travail. Le problème est de déterminer les traits de la situation effectivement pris en compte par le sujet à un moment donné de la constitution et du fonctionnement de ces automatismes. La méconnaissance de ces traits et de leur pertinence est en effet génératrice de l'un ou l'autre de ces deux types d'erreurs : erreurs déclenchées dans des conditions où l'automatisme n'est pas pertinent (parce que cette situation a été indûment assimilée à une situation où l'automatisme est valable) ou erreurs résultant du non- déclenchement de l'automatisme là où il serait nécessaire (parce que la situation a été jugée non pertinente pour l'utilisation de l'automatisme).

Il ne faut pas oublier que si la condition d'uniformité des conditions d'exécution d'une exécution à l'autre est importante, cette uniformité ne peut se déduire simplement des conditions objectivement décrites, mais qu'elle est liée à la représentation que se fait le sujet des conditions dans lesquelles cet automatisme trouve place. Cette question devient capitale dans les situations de transfert pour déterminer les conditions effectivement prises en compte dans le processus d'automatisation.

Il a été noté aussi qu'une certaine variabilité des conditions d'exécution, parfois inévitable, comportait éventuellement des avantages en ce sens qu'elle pouvait maintenir un certain caractère adaptatif aux automatismes. Ainsi, Falzon et Teiger (1995) ont noté que « la fonction constructive de la répétition tire parti des variations de l'environnement qui conduisent à la construction de schèmes adaptatifs » (p. 35).

\subsection{3.- L'électivité progressive}

Cette expression empruntée à Wallon (1982/38) souligne l'idée que les éléments qui constituent un automatisme sont initialement empruntés à des ensembles préalablement associés et, ensuite, progressivement intégrés dans un nouvel ensemble. Dans un texte où il traite des automatismes sensorimoteurs, il écrit ainsi : « Acquérir un automatisme, ce n'est pas enchaîner dans un ordre invariable des gestes sélectionnés une fois pour toutes. C'est en un sens faire exactement l'inverse. C'est dissoudre des blocs préexistants de mouvements afin de n'utiliser que les combinaisons requises par l'acte en cours d'exécution (...). Les syncinésies doivent être dissociées, les gestes superflus ou gênants inhibés. Que les automatismes soient naturels, comme la marche et la préhension, ou qu'ils 
répondent à des techniques apprises, comme la danse, l'écriture, le jeu de pianiste, leur agilité est toujours liée au pouvoir de supprimer toutes les contractions parasites » (p. 235).

La constitution des automatismes s'accompagne souvent, initialement, de la déstructuration des actions complexes à partir desquelles ils ont été construits. Mais une fois que les éléments résultants sont insérés dans l'action qui va progressivement s'automatiser, ils sont transformés par cette intégration à l'automatisme, ce dont rend bien compte la théorie de l'activité de Leontiev (1972, 1975), comme on le verra ci-dessous. Ceci explique pourquoi il est très difficile de modifier un automatisme : en soustraire ou en transformer un élément, c'est l'altérer et lui faire perdre ses propriétés à un degré souvent important, avec le risque de retour à l'automatisme primitif. L'électivité peut être aussi conçue comme l'élimination progressive de composantes de l'action initiale qui s'avèrent non pertinentes pour l'automatisme. C'est à quoi invitent les recherches de Lee et Anderson (2001) et celles qu'ils rappellent dans leur texte. Etudiant notamment l'évolution des lieux du champ fixés au cours de l'apprentissage d'une tâche, ils montrent que cet apprentissage s'accompagne d'une réduction des fixations oculaires sur des éléments du champ apportant une information non pertinente à la tâche, sans que les opérateurs soient conscients de ce phénomène. En extrapolant (avec prudence) ces résultats, on pourrait concevoir l'acquisition de l'automatisme comme une focalisation sur les parties les plus pertinentes du champ de la tâche, focalisation corrélative d'un rétrécissement de la connaissance par l'opérateur du contexte de son action.

\subsection{4.- Des effets négatifs des avantages des automatismes}

Quoique ce texte veuille essentiellement mettre en lumière les avantages des automatismes, il ne peut ignorer les effets négatifs auxquels peuvent conduire certains de ces avantages. Ainsi l'électivité progressive qui vient d'être décrite peut conduire à un rétrécissement du champ et des possibilités d'adaptation des automatismes, rétrécissement décrit plus haut (2.2.) au titre de l'absence de contrôle attentionnel. Cette focalisation peut entraîner la négligence d'autres traits qui sont des conditions de validité de la réponse. Quand certaines conditions changent, le sujet continue alors à déclencher l'automatisme parce que le trait habituellement pertinent est présent, mais avec d'autres qui devraient l'invalider.

Les effets de ce rétrécissement et de cette rigidification des automatismes s'exerçant longtemps dans un champ étroit ont été signalés par Teiger, Laville, Dessors et Gadbois(1977) à propos des opératrices de renseignements téléphoniques. Nous les avions antérieurement cités (Leplat, 1988) dans un passage qui sera partiellement rappelé ici : "Certaines situations de la vie quotidienne, dès lors qu'elles ont quelque similitude avec la situation de travail, déclenchent automatiquement les comportements stéréotypés imposés par l'activité professionnelle (...). C'est ainsi que les opératrices se surprennent souvent à répondre « allo » au lieu de « comment » dans les situations les plus diverses, aux repas, chez l'épicier ou chez le coiffeur » (p. 65-6) ou encore à répéter la formule d'accueil au téléphone en entendant la sonnerie de fermeture des portes du métro. On verra plus loin comment réduire ces effets négatifs des automatismes en évitant de laisser ceux-ci se fermer sur eux-mêmes.

\section{2.- Un essai de classification des automatismes}

Comme les pages précédentes ont permis de le constater, les automatismes sont des objets complexes, insérables dans des réseaux divers, et donc susceptibles de classifications fondées sur des principes variés. Nous proposons ici une classification établie sur un principe simple, à partir d'un modèle très élémentaire de l'activité, exposé ailleurs en détail (Leplat, 1997), mais dont l'essentiel seulement sera retenu ici. Trois éléments figurent dans ce modèle : l'activité, le sujet (conditions internes de l'activité) et la tâche (conditions externes de l'activité). Le schéma de la figure 1 fait apparaître quelques relations typiques entre ces trois catégories d'éléments. L'activité et ses composantes dont l'automatisme est celle qui nous intéresse plus directement ici dépendent des conditions 
externes (techniques, organisationnelles, etc.) (flèche 1) que l'activité contribue à modifier (flèche 2). L'activité dépend aussi des conditions internes caractéristiques du sujet, en particulier des compétences (flèche 3 ) et son exécution a des conséquences sur ces conditions (flèche 4). L'activité se traduit par deux phénomènes importants illustrés par les flèches pointillées de ce schéma. Le premier est l'intériorisation de la tâche par le sujet, le second est l'extériorisation de certains traits du sujet, ceux justement qui sont mis en œuvre par l'activité (par exemple, sa compétence).

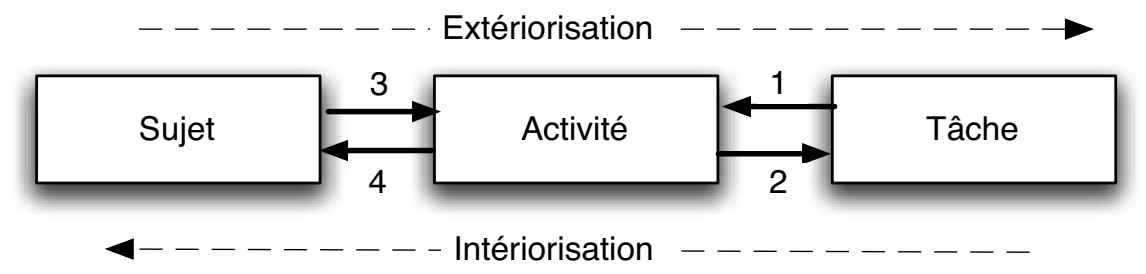

Figure 1.- . Schéma simplifié des conditions de l'activité

1. L'activité dépend de la tâche ; 2. L'activité a des conséquences sur la tâche ;

3. L'activité dépend du sujet ; 4. L'activité a des conséquences pour le sujet.

L'activité participe au double mouvement d'intériorisation des exigences de la tâche et d'extériorisation des caractéristiques du sujet (par exemple, de ses compétences).

Analyser un automatisme consistera à identifier la tâche à laquelle il correspond et les caractéristiques du sujet, ici, les compétences, mises en jeu dans l'exécution de celle-ci. Pour l'illustration sommaire que nous voulons donner, trois niveaux de tâche seront retenus. Il ne faut surtout pas y voir une esquisse de typologie : les niveaux ont été choisis essentiellement pour illustrer une démarche d'analyse des automatismes. A chacun de ces niveaux de tâche, on peut faire correspondre des caractéristiques du sujet qui les exécute : on a choisi ici de se focaliser sur les compétences, systèmes de connaissances engendrant l'activité. Ces compétences prennent parfois des noms divers selon les niveaux et les modèles privilégiés par l'analyste. On peut voir les automatismes comme une forme de compétences, comme un stade avancé du développement de celles-ci. Le tableau 1 illustre sommairement l'articulation de toutes ces notions : on en donnera un bref commentaire. (Le stade ultime des automatismes est noté au terme du pointillé, avec la flèche - ->).

\begin{tabular}{|c|c|}
\hline $\begin{array}{c}\text { Versant tâche } \\
\text { (niveaux et exemples) }\end{array}$ & $\begin{array}{c}\text { Versant sujet } \\
\text { (de la compétence à l'automatisme) }\end{array}$ \\
\hline $\begin{array}{l}\text { 1. Tâche étroitement définie (prescription, } \\
\text { instruction, etc.) } \\
\text { Ex.: tâches à cycle court dans le travail à } \\
\text { la chaîne }\end{array}$ & $\begin{array}{l}\text { Schème, script, compétences incorporées } \\
\qquad--\rightarrow \rightarrow \text { stéréotype }\end{array}$ \\
\hline $\begin{array}{l}\text { 2. Tâches à large champ, avec } \\
\text { incertitude. } \\
\text { Ex.: réparation d'un engin, conduite d'un } \\
\text { dispositif complexe }\end{array}$ & $\begin{array}{c}\text { Compétences plus ou moins incorporées } \\
\qquad---\rightarrow \text { routines }\end{array}$ \\
\hline $\begin{array}{l}\text { 3. Tâches mettant en jeu l'environnement } \\
\text { socio-technique } \\
\text { Ex.: tâches collectives, négociation d'un } \\
\text { contrat }\end{array}$ & $\begin{array}{c}\text { Compétences sociales } \\
--->\text { habitus, genre, coutume }\end{array}$ \\
\hline
\end{tabular}

Tableau 1.-Analyse des conditions propres à caractériser les automatismes 
- Niveau 1. A une tâche étroitement définie correspond une compétence désignée, selon le cadre de référence de l'analyste, comme schème (Vergnaud, 1990 ; Rabardel, 1995) ou comme script (Schank \& Abelson, 1997) ou comme compétence incorporée (Leplat, 1997). L'automatisme peut être vu comme une étape avancée du développement de la compétence. Une étape ultime serait celle du stéréotype dans laquelle la compétence se ferme en quelque sorte sur elle-même et perd progressivement son adaptabilité.

- Niveau 2. Les compétences correspondant à ce type de tâches sont parfois désignées aussi avec les termes du niveau 1, mais on leur laisse aussi le terme de compétence. Elles peuvent être plus ou moins incorporées et leur stade ultime est celui de routine qui a les traits du stéréotype. Les compétences de ce niveau peuvent être souvent considérées comme l'intériorisation de techniques (Leplat \& Pailhous, 1981/2002). Au stade extrême, on trouverait les routines qui ont pu être définies comme des « modèles d'activité humaine qui assurent ainsi la cohérence des décisions individuelles » (Reynaud, 1998, p. 475) ; certains de leurs aspects les rangent parfois au niveau suivant.

- Niveau 3. Les tâches correspondant à ce niveau sont à la fois variées, plus étendues et moins bien définies. Il s'agit souvent d'un complexe de tâches. Les compétences correspondantes sont de nature sociale, et quand elles sont bien incorporées, elles sont désignées sous les noms d'habitus, genre, coutume. Bourdieu (1980) en a donné de nombreuses définitions et commentaires. Retenons celui-ci : «Produit de l'histoire, l'habitus produit des pratiques, individuelles et collectives, donc de l'histoire, conformément aux schèmes engendrés par l'histoire ; il assure la présence active des expériences passées qui, déposées en chaque organisme sous la forme de schèmes de perception, de pensée et d'action, tendent plus sûrement que toutes les règles formelles et toutes les normes explicites, à garantir la conformité des pratiques et leur constance à travers le temps » (p. 91). On peut rapprocher de la notion d'habitus celle de genre que Clot (1999) et Clot, Faïta, Fernandez et Scheller (2001) définissent comme «une sorte d'intercalaire social», «une sorte de mémoire mobilisée par l'action » qui « donne sa contenance à l'activité en situation : manière de se tenir, manière de s'adresser, manière de commencer une activité et de la finir, manière de la conduire efficacement à son objet » (2001, p. 19)

Cet essai de classification des automatismes, qui suppose familier un certain nombre de connaissances de psychologie ergonomique, aurait mérité une place plus grande qui ne pouvait lui être accordée étant donnée l'organisation de ce texte. Tel quel, il propose des pistes pour l'analyse des automatismes et il suggère des rapprochements entre différentes sortes d'automatismes qui peuvent être utiles si l'on sait se garder d'assimilation trop rapides.

\section{3.- Les automatismes dans les modèles de l'activité}

Les analyses précédentes auront montré que les automatismes sont des formes ou des composantes de l'activité. Il est donc utile d'étudier comment ils trouvent leur place dans différents modèles ou théories de l'activité. Une telle démarche devrait être révélatrice de caractéristiques des automatismes et elle devrait mettre en lumière certaines de leurs facettes. Comme le remarque Logan (1988) en comparant sa théorie à d'autres, «je ne cherche pas à montrer que cette théorie est plus correcte et plus exacte que les autres. Je considère plutôt les théories comme abordant différentes situations, et des théories peuvent convenir à certaines situations plus qu'à d'autres. Il me semble vraisemblable que les hommes peuvent apprendre de plus d'une manière. Le choix entre des théories est quelque chose qui ressemble au choix entre tests statistiques » (p. 517). Le choix des modèles qui a été fait ici est évidemment partiel et partial : il a privilégié les modèles qui sont parmi les plus fréquemment évoqués en ergonomie et en psychologie du travail. Ces modèles ne sont pas tous du même type et n'ont surtout pas à être conçus comme concurrents. Ils sont à considérer comme des vues pri- 
ses sur cette entité complexe qu'est l'activité, entité qui déborde toujours les modèles cherchant à la formaliser. D'où l'intérêt de ces vues multiples dont la pertinence est liée aussi à l'objet de l'étude dans laquelle ils s'inscrivent. On commencera par exposer des modèles mettant l'accent sur les modes élémentaires de fonctionnement, puis sur des modèles mettant l'accent sur la genèse du fonctionnement automatique, enfin, on terminera par la présentation de modèles mettant l'accent sur le rôle du couplage.

\section{1.- Les modèles mettant l'accent sur les fonctionnements élémentaires}

On mentionnera ici trois catégories de modèles développés dans le cadre de la psychologie cognitive, et qui ont été particulièrement marquantes dans ce domaine. Les deux premières, notamment, ont inspiré beaucoup de travaux expérimentaux. Mais toutes peuvent éclairer les analyses des automatismes en situation de travail.

\subsection{1.- Un modèle de l'automatisme fondé sur la récupération de cas en mémoire}

Ce modèle, développé par Logan (1988), est dit par cet auteur «théorie des cas » («instance theory of automatization»). Cette théorie qui fait jouer un rôle majeur à la mémoire implique un mécanisme d'apprentissage fondé sur «l'accumulation de traces épisodiques discrètes avec l'expérience » (p.493), c'est-à-dire de cas qui pourront être ultérieurement récupérés ( «automaticity is memory retrieval », p. 493) : d'où le nom de théorie des cas qui lui est aussi donné. Elle suppose que les novices commencent avec des instructions (il parle d'algorithme) suffisantes pour exécuter la tâche. Avec la pratique, «ils apprennent des solutions spécifiques à des problèmes spécifiques, solutions qu'ils récupèrent quand ils rencontrent les mêmes problèmes. Alors, ils ont le choix de répondre avec la solution récupérée en mémoire ou avec celle calculée à partir de l'algorithme » (id.). Quand ils ont acquis assez d'expérience pour répondre avec une solution issue de la mémoire, ils peuvent abandonner complètement les instructions. C'est alors que leur performance est automatique. L'automatisation exprime le passage d'une activité fondée sur les instructions à une activité fondée sur la mémoire. Logan montre que sa théorie est compatible avec les propriétés des automatismes classiquement reconnues (réduction du temps d'exécution, autonomie, etc.). Cette théorie implique donc un double type de fonctionnement fondé sur des mécanismes différents, « item-based / process-based».

\subsection{2.- Le modèle de Schneider et Shiffrin}

Ce modèle a été conçu et mis en oeuvre en psychologie expérimentale à partir d'un ensemble d'expériences conduites par Schneider et Shiffrin (1977), Shiffrin et Schneider (1977), sur des tâches simples, en particulier de détection, expériences comportant entre 2000 et 12000 essais par sujet. L'un de ces auteurs et un associé (Schneider \& Chein, 2003), 25 ans après, ont présenté une synthèse des recherches entreprises depuis concernant la distinction et les caractéristiques des deux types de traitement, contrôlé et automatique. Ils en proposent une formulation plus précise en même temps qu'ils en examinent les fondements neuro-anatomiques. Ils soulignent aussi l'avantage apporté par ce double système de traitement.

On trouvera dans un chapitre de Camus (1988) un exposé et des commentaires détaillés des expériences rapportées par les auteurs précédents. On retiendra quelques conclusions dégagées par ces auteurs de leur ensemble d'expériences en ce qui concerne les deux processus ("processings ») qu'ils ont étudiés, contrôlé et automatique (Shiffrin \& Schneider, 1977, p. 160 sq.).

Les processus contrôlés sont : a) des processus à capacité limitée, requérant l'attention, qui opèrent en série et non en parallèle. b) Leurs limitations sont celles de la mémoire à court terme, notamment la quantité limitée d'information qui peut être conservée sans perte. c) Ces processus peuvent être 
rapidement mis en œuvre et aisément modifiés, souvent à partir d'instructions verbales. d) Ils « peuvent contrôler le flux des informations à l'intérieur et entre les niveaux, et entre les mémoires à court et à long terme ». e) « ils se développent rapidement pour atteindre une performance asymptotique (...). Quand la performance s'améliore avec les essais, elle le doit au changement du processus de contrôle ou au développement du contrôle automatique » (p. 160) ou par des altérations de l'organisation du processus de contrôle par un long apprentissage.

Les processus automatisés «a) ne sont pas gênés par les limitations de la capacité de la mémoire à court terme et n'exigent pas d'attention. Ainsi, ils apparaissent souvent agir en parallèle et quelquefois indépendamment l'un de l'autre. b) Certains peuvent être déclenchés sous le contrôle du sujet, mais une fois déclenchés, ils se déroulent automatiquement (...). c) Leur développement exige un entraînement considérable et une fois appris, ils sont difficiles à modifier. d) Leur vitesse et leur automaticité tiennent habituellement leurs éléments constituants hors de la perception consciente » (p. 160).

La combinaison des processus automatiques et contrôlés. "Quoique les entrées sensorielles soient d'abord codées avec un système de traitement automatisé, les résultats étant rendus disponibles pour le processus contrôlé, il ne doit pas être conclu que le processus automatique précède invariablement le processus contrôlé. En fait, les deux processus peuvent progresser en parallèle (...). Et même plus important, le processus contrôlé est souvent utilisé pour mettre en route le processus automatique. En particulier, les situations complexes peuvent exploiter des mélanges de processus contrôlés et automatisés » (p. 161). Ce sera souvent un objectif important de l'analyse de l'activité que d'étudier comment s'élaborent et s'articulent les deux processus.

\subsection{3.- Des modèles du fonctionnement automatique dans des activités complexes}

L'existence de deux modes de fonctionnement a été reconnue depuis longtemps dans l'étude des tâches complexes, comme l'histoire de la psychologie en témoigne. Janet (1886) l'évoque en citant un long passage de Condillac (1798) qui commence ainsi : «il y a en quelque sorte deux moi dans chaque homme : le moi d'habitude et le moi de réflexion. (...) Le premier dirige toutes les facultés animales (...), le second est excité par toutes sortes de choses qui, en nous donnant de la curiosité, nous porte à multiplier nos besoins » (p. 435). L'habitude, dans un tel texte se rapproche de ce que nous appellerions automatisme.

Revenons à notre époque! Les modèles du fonctionnement cognitif dans l'exécution des tâches complexes sont largement répandus et font souvent place à l'automatisme. Celui de Norman (1988) retient deux modes de fonctionnement cognitif : «Il y a plusieurs modes de cognition, plusieurs voies différentes ouvertes à la pensée. Les deux modes particulièrement pertinents à mon analyse sont dits "cognition par l'expérience" ("experiential cognition") et "cognition par la réflexion" ("reflective cognition") » (Norman, 1993, p. 16). Les automatismes entrent dans le premier mode de fonctionnement dont ils partagent beaucoup de traits. Ils sont dirigés par les données (« datadriven »). Ils correspondent à un traitement de mode réactif qui «met en jeu une information qui existe déjà en mémoire et qui est simplement réactivée»(p. 24). Le fonctionnement à base de réflexion, lui, prend du temps, il requiert l'aptitude à stocker temporairement des résultats, à faire des inférences à partir de l'information stockée, et à suivre des chaînes de raisonnement en arrière et en avant» (p. 25). Il est dirigé par des concepts («conceptually driven»), de type «top-down». Norman note à juste titre que : «Ces deux modes de cognition ne capturent pas le tout de la pensée, ni ne sont complètement indépendants : il est possible d'en avoir un mélange, profitant du mode à base d'expérience tout en réfléchissant simultanément sur lui » (p. 16). On retrouve ainsi la distinction des auteurs précédents entre processus, contrôlé et automatisé, distinction cruciale dont les termes ne sont pas seulement à différencier, mais aussi à articuler. 
Des auteurs qui ont proposé ce double mode de fonctionnement, on peut retenir quelques conclusions. 1) Aucun des deux modes ne peut être déclaré en soi supérieur à l'autre : ils sont tous les deux nécessaires et ils ont des fonctions différentes dans l'activité. Ils répondent à des situations différentes et comme le souligne Norman (1993), le danger est qu'ils soient employés l'un pour l'autre. 2) Cette distinction permet d' « éclairer et de comparer différents aspects de l'activité mentale » (Norman, 1993, p. 16). 3) Ces deux modes ne sont pas indépendants et leur place n'est pas invariable dans une même tâche. Elle varie avec le niveau d'apprentissage et avec les contraintes de la tâche.

Comme le note justement Norman (1993, p. 15), «il y a plusieurs modes de cognition » qui ne se résument pas aux deux précédents ». Ainsi, le modèle de Rasmussen (par exemple, Rasmussen, Pejtersen, \& Goodstein, 1994), bien connu des ergonomes, comporte trois niveaux dont le niveau dit «skill based » peut être considéré comme celui de l'automatisme.

\section{2.- Les modèles mettant l'accent sur la genèse du fonctionnement automatique}

Les modèles présentés dans cette catégorie ont accordé une importance particulière à la manière dont les automatismes s'élaborent et se mettent en place. On en retiendra deux types : le premier s'inscrit dans une perspective cognitiviste orientée vers l'analyse des habiletés, le second est issu du courant de recherche dénommé souvent théorie de l'activité, initialement développé en Russie et qui a ensuite été largement exploité dans les recherches psychologiques et ergonomiques menées dans le domaine du travail.

\subsection{1.- La genèse des automatismes dans la perspective de l'acquisition des habiletés}

Dans le système ACT bien connu en psychologie cognitive, l'automatisation des activités cognitives fait intervenir une étape à l'intérieur du processus de compilation, dite de composition. L'idée de base en est que des règles de production «qui sont exécutées initialement en séquences sont progressivement combinées en règles de production simples » (Neves \& Anderson, 1981, p. 67), moins nombreuses, ce qui contribue à réduire les temps d'exécution de la procédure globale. Voici un exemple inspiré de ceux proposés par les auteurs :

- Règle 1 : Si vous voyez une lumière rouge, Alors, il y a un danger.

- Règle 2: S'il y a un danger et qu'une personne soit près de vous, Alors, avertir la personne.

- Dans certains cas, ces deux règles peuvent être composées. On aura alors :

- Règle 3 : Si vous voyez une lumière rouge et qu'une personne est près de vous, Alors, il y a un danger et avertir la personne.

Ce mécanisme d'agrégation de règles permet d'expliquer des propriétés des automatismes. Il fournit une interprétation de la réorganisation de l'activité. C'est ainsi que Cheng (1985) discutant des travaux expérimentaux sur l'automatisme a pu écrire que : « l'amélioration de la performance (...) peut être due à une restructuration des composantes de la tâche qui coordonne ces dernières, les intègre, les réorganise en nouvelles unités perceptives, cognitives, motrices » (p. 414). Ce mécanisme de composition permet aussi de comprendre le phénomène de continuation (cf. 1.3) comme la difficulté de modifier un automatisme constitué. « Le résultat de la composition est de transformer une procédure conditionnelle ouverte en une procédure inconditionnelle fermée. Par exemple, quand une production est créée à partir de la composition de cinq autres, elle fait du travail de ces cinq productions une étape qui ne peut être interrompue. S'il y avait une autre production à insérer dans le milieu de cette séquence à cinq étapes, elle ne pourrait l'être car elle serait contournée » (Neves et Anderson, 1981, p. 73). 


\subsection{2.- L'automatisme dans le modèle de l'activité issu de la psychologie russe}

Ce modèle initialement présenté par Leontiev $(1972,1975)$ et récemment commenté par Bedny et Karwowski (2004) a largement influencé les analyses du travail conduites en ergonomie et en psychologie du travail. Dans ce modèle, l'activité, caractérisée par son motif, à « fonction incitative », se réalise par des actions, «processus soumis à la représentation du résultat qui doit être atteint, c'est-à-dire un processus soumis à un but conscient » (Léontiev, 1975, p. 113). L'action est réalisée par des opérations : «j'appelle opérations les moyens d'accomplissement de l'action » (p. 118). Ces opérations dépendent des conditions dans lesquelles le but de l'action doit être atteint. Par exemple, pour aller à son lieu de travail (action), on pourra utiliser des trajets différents (opérations) selon l'état météorologique, la circulation, les contraintes de temps. Le phénomène très important souligné dans cette théorie, c'est que « toute opération résulte d'une transformation de l'action procédant de son incorporation dans une autre action et de sa "technicisation" consécutive » (p. 119). Léontiev illustre ce phénomène par un bon exemple issu de la conduite automobile que nous avons déjà eu l'occasion de citer. Prenons-en un autre. Quand on acquiert un nouveau magnétoscope, son usage est réglé par une suite d'actions définies sur la notice. Ainsi, pour programmer un enregistrement, la succession d'actions suivantes est requise : mettre la cassette en place, ... fixer l'horaire, ... choisir la source souhaitée, etc. Après un usage répété, ces actions prennent le statut d'opérations et ne sont plus exécutées comme orientées par leur but primitif, mais comme orientées par le but de l'action plus globale (mettre le dispositif en état d'enregistrement) à laquelle elles sont intégrées. Léontiev (1975) souligne justement que "l'opération n'est quand même jamais "coupée" de l'action comme l'action n'est jamais “coupée” de l'activité » (p. 120).

La notion d'automatisme peut être rapprochée de celle d'opération et bien des analyses concernant la notion d'opération valent aussi pour celle d'automatisme. Ainsi, dans la transformation de l'action en opération, la coordination des opérations à l'intérieur de la nouvelle action s'automatise, elle aussi, en même temps que les opérations. Dans cette transformation de l'action en opération, cette dernière s'intègre dans une nouvelle action qui l'englobe (mettre le dispositif en marche dans l'exemple précédent). Cette intégration n'est pas une simple juxtaposition. La nouvelle action s'accompagne d'un remodelage des éléments qui la constituent. Le passage au statut d'opération, puis d'automatisme, ne laisse donc pas inchangées les actions dont le statut s'est modifié. On s'en aperçoit quand on veut réutiliser cette opération pour la joindre à une autre action. Wallon (1938) avait bien vu ce problème quand il déclare qu' «acquérir un automatisme (...), c'est dissoudre les blocs préexistants de mouvements, afin de n'utiliser que les combinaisons requises par l'acte en cours d'exécution » (p. 235). La répétition entraîne ainsi une transformation des composantes de l'action dont l'automatisation est un aspect essentiel, mais non unique.

L'exploitation de la théorie de l'activité pour l'analyse de l'exécution de tâches complexes conduit à étudier les mécanismes de leur apprentissage, en particulier comment les actions plus globales «opérationnalisent» les actions élémentaires. C'est dans cette perspective que Galpérine (1966) s'est spécialement intéressé à la «formation par étape des actions ». Il déclare que « la formation d'une action nouvelle (et à plus forte raison d'une opération mentale) ne se fait jamais momentanément < lisons plutôt instantanément>. Il s'agit d'un processus où l'on distingue des phases fondamentales et, à l'intérieur de chacune, des changements plus fugitifs » (p. 117). Au début, «l'action se déploie et se réalise au ralenti », puis un processus dit de "généralisation » vise à « séparer l'essentiel du non essentiel », c'est-à-dire à dégager un «contenu permanent » (on aurait envie de dire des invariants). «Cette séparation du contenu permanent et dans ce sens stéréotypé... » (p. 120) aboutit à ce que Galpérine appelle la réduction de l'action. Dans ces analyses, l'accent est mis sur les actions, mais non sur l'évolution des opérations. Ainsi, la notion d'automatisation n'apparaît pas directement : on peut seulement la supposer sous le terme de «stéréotypé ». Mais Galpérine travaillait sur des enfants et s'intéressait à l'acquisition proprement dite plus qu'au développement de l'action une fois constituée. 
Chez Léontiev (1972) apparaît un peu plus nettement le rapprochement entre opération et automatisme quand il déclare : "Comment une action se transforme-t-elle en opération et, par conséquent, en savoir-faire et en habitude ?» (p. 296). Il mentionne plus loin, «... les opérations mentales et leur fixation sous forme d'habitudes mentales» (p. 297). Il note aussi qu'en devenant opération l'action antérieure est non conscientisée, ce qui la rapproche de l'automatisme, mais qu'elle peut l'être à nouveau, c'est-à-dire repasser à l'état d'action. Léontiev (1975) fait apparaître un autre trait qui rapproche les opérations des automatismes en remarquant leur caractère autonome qui les rend exécutables par « un mécanisme automatique. En général, le sort des opérations est, tôt ou tard, de devenir des fonctions de la machine » (p. 120).

\section{3.- L'automatisme dans les théories mettant l'accent sur le rôle du couplage entre le sujet et la tâche}

Le rôle de ce couplage entre conditions internes et externes ne peut être ignoré dans aucune théorie, mais dans certaines, il est particulièrement crucial, en particulier dans les théories de l'action située, de la cognition distribuée et de l'énaction. Le couplage est conçu de manière différente dans ces théories mais on se contentera ici d'en donner quelques formulations qui peuvent être mises plus directement en rapport avec les automatismes.

Sur le plan cognitif, Norman (1988) a présenté les caractères du couplage dans un chapitre intitulé «Les connaissances dans la tête et dans le monde.» «Les connaissances dans le monde agissent comme leur propre organe de rappel. Elles peuvent nous aider à récupérer des structures que nous aurions oubliées autrement. Les connaissances dans la tête sont efficientes : aucune recherche ni interprétation de l'environnement n'est exigée. Pour utiliser les connaissances dans la tête, nous avons d'abord à les y mettre, ce qui peut exiger des quantités considérables d'apprentissage. Les connaissances dans le monde sont plus faciles à acquérir, mais souvent plus difficiles à utiliser. Et elles reposent lourdement sur la présence physique continue de l'information ; que l'environnement change et l'information est changée. La performance repose sur la présence physique de l'environnement de la tâche » (p. 80). Il note que « dans les situations de la vie quotidienne, le comportement est déterminé par la combinaison de connaissances internes et de connaissances externes » dont il découle que «les gens peuvent délibérément organiser l'environnement pour assister leur comportement » (p. 55). Ainsi, l'exécution d'une tâche peut être réalisée avec une répartition différente des connaissances dans la tête et dans le monde. Dans une perspective ergonomique, l'accent est mis sur les connaissances externes, c'est-à-dire sur l'aménagement de l'environnement qui permet de réduire les exigences du travail pour l'opérateur. C'est une autre manière de dire que dans ce cas l'activité est guidée par des automatismes antérieurement acquis par les opérateurs ou même que ces automatismes ont été pris en charge par des automatismes techniques (par exemple, la machine est automatiquement débranchée en cas de manoeuvre erronée).

La connaissance dans le monde s'exprime par la notion d'affordance des objets. Ce terme « se rapporte aux propriétés perçues et présentes de la chose, d'abord à ces propriétés fondamentales qui déterminent seulement comment la chose pourrait être utilisée » (Norman, 1988, p. 9). Ainsi, une chaise appelle l'acte de s'asseoir : on sait s'en servir, dans notre société, sans qu'il soit besoin d'instruction particulière et sans avoir recours à une représentation. On rejoint ainsi, par une autre voie, des analyses de Merleau-Ponty sur les rapports entre conditions internes et conditions externes: «Puisque tous les mouvements de l'organisme sont toujours conditionnés par des influences externes, on peut bien, si l'on veut, traiter le comportement comme un effet du milieu. Mais de la même façon, comme toutes les stimulations que l'organisme reçoit n'ont à leur tour été possibles que par ses mouvements précédents, qui ont fini par exposer l'organe récepteur aux influences externes, on pourrait dire aussi que le comportement est la cause première de toutes les stimulations. Ainsi la forme de l'excitant est créée par l'organisme lui-même, par sa manière propre de s'offrir aux actions 
du dehors » (p. 9). « Avant toute interprétation systématique, la description des faits connus montre que le sort d'une excitation est déterminé par son rapport à l'ensemble de l'état organique et aux excitations simultanées ou précédentes, et qu'entre l'organisme et son milieu, les rapports ne sont pas de causalité linéaire, mais de causalité circulaire » (p. 12). On peut lire une définition de l'automatisme moteur et de son acquisition dans la phrase suivante : "Un mouvement est appris lorsque le corps l'a compris, c'est-à-dire lorsqu'il l'a incorporé à son 'monde', et mouvoir son corps, c'est viser à travers lui les choses, c'est le laisser répondre à leur sollicitation qui s'exerce sur lui sans aucune représentation » (1945, p. 161).

La notion de couplage a été développée et approfondie dans les théories de l'énaction qui reconnaissent leur parenté avec la perspective exposée dans des textes de Merleau-Ponty qui viennent d'être rappelés. Ce dernier rattachait aussi ses conceptions à celles développées par Weizsäcker qui écrivait que «l'organisme justement ne peut être comparé à un clavier sur lequel joueraient les stimuli extérieurs et où ils dessineraient leur forme propre pour cette simple raison qu'il contribue à le constituer» (cité par Merleau-Ponty, 1942, p. 9). On trouve des déclarations très voisines chez Varela (1989) : «La perception et l'action ne peuvent être séparées parce que la perception exprime la clôture du système nerveux (en note : comme le dit si bien Hanson : "ce sont les gens et non leurs yeux qui voient. Les cameras et les yeux sont aveugles" (1958, p. 9). En termes plus positifs, "percevoir équivaut à construire des invariants par un couplage sensori-moteur qui permet à l'organisme de survivre dans son environnement » (p. 154). Cette notion de couplage structurel se généralise au domaine de la cognition. Winograd et Flores (1989) rapprochant les vues précédentes de celles de Heidegger parlent du fonctionnement humain comme celui de «systèmes structurés et déterminés, façonnés par le couplage structurel. Chaque organisme est engagé dans un mode d'activité qui est déclenché par des changements de milieu et qui a la possibilité de changer la structure de l'organisme (et partant de changer son comportement futur)»(p.121). Dans cette conception, l'environnement change de statut : il n'est plus une entité externe dotée de propriétés fixées, car le couplage transforme les deux termes qui le constituent.

On trouve, dans le langage phénoménologique de Merleau-Ponty (1945), des analyses et des formulations très suggestives de ces phénomènes. Retenons, en particulier, cette déclaration: «l'expérience motrice de notre corps n'est pas un cas particulier de connaissance, elle nous fournit une manière d'accéder au monde et à l'objet, une "praktognosie" (en note, Grünbaum, Aphasie und Motorik) qui doit être reconnue comme originale et peut-être comme originaire. Mon corps a son monde ou comprend son monde sans avoir à passer par des "représentation"”, sans se subordonner à une "fonction symbolique" ou "objectivant" » (p. 164). Les exemples qui sont donnés de cette conception sont très riches et suggestifs, en particulier, celui de l'organiste (p.169, sq.).

Si l'automatisme est conçu, non comme une structure figée, non comme un stéréotype, qui serait pure répétition à l'identique, mais comme disposant d'un pouvoir d'adaptation plus ou moins étendu, il pourrait être dit de l'automatisme ce que Merleau-Ponty (1945) dit de l'habitude : «c'est le corps, comme on l'a dit souvent, qui "attrape" ("Kapiert") et qui "comprend" le mouvement» (p. 167) ; l'habitude « est un savoir qui est dans les mains et qui ne se livre qu'à l'effort corporel et ne peut se traduire par sa désignation objective» (p. 168). On retrouve ici l'idée de l'automatisme comme compétence incorporée, laquelle s'exprime dans l'action.

\section{4.- Pour un bon usage des automatismes en situation de travail}

Les automatismes ont une large place dans les activités en situation de travail. Une pratique prolongée favorise la constitution d'automatismes couvrant, selon les cas, un champ plus ou moins large. La psychologie du travail et l'ergonomie n'ignorent pas ces phénomènes, mais elles en ont surtout mis en lumière les conséquences négatives. L'exemple le plus typique en est fourni par les études de 
l'erreur et de la fiabilité qui ont identifié les types d'erreurs dans la genèse desquels les automatismes étaient en jeu. Ce serait une longue partie que nécessiterait l'actualisation de cet inventaire. Nous avons choisi ici d'examiner plutôt les aspects positifs des automatismes et de le faire en essayant de dégager quelques recommandations propres à la mise en valeur de ces aspects positifs. On pourra lire aussi à travers ces recommandations quelques pistes possibles de recherche qui aideraient à les préciser et à les généraliser.

\section{1.- Rappel des avantages des automatismes}

L'examen des propriétés des automatismes a fait apparaître quelques-unes de leurs qualités : on rappellera ici celles qui sont plus directement liées à la faiblesse du coût cognitif ou charge mentale qu'ils entraînent.

- Possibilité de fonctionnement en parallèle. Les automatismes peuvent fonctionner en parallèle avec des activités contrôlées ou d'autres automatismes. Quand le conducteur automobile a automatisé une partie importante des composantes de sa conduite, il reste disponible, dans des conditions « ordinaires » pour d'autres activités : poursuivre une conversation, anticiper des situations critiques, être plus attentif à des changements de l'environnement, etc. Mais quand ces activités deviennent centrales et que la situation de conduite demande réflexion, alors un incident peut se produire (d'où l'interdiction de l'usage du téléphone dans la conduite automobile).

Les tâches en temps partagé souvent signalées dans les travaux modernes sont plus faciles à traiter quand l'une d'elles est plus ou moins automatisée : c'est le cas, par exemple de l'attention flottante qui a été repérée dans certains travaux au cours desquels l'opérateur saisit, dans les échanges verbaux qui ont lieu dans son environnement, des informations qui ne lui sont pas destinées, mais qui peuvent être utiles à la gestion personnelle ou collective du travail (Dusire, 2000 ; Rognin, Salembier, \& Zouinar, 2000). L'automatisation de l'activité principale favorise cette écoute.

- Résistance aux facteurs perturbateurs : les automatismes sont moins sensibles que les activités contrôlées aux perturbations des conditions d'ambiance (bruit, chaleur, conversations parasites, etc.). Il existe cependant une perturbation à laquelle les automatismes sont sensibles qui est l'interruption en cours d'exécution. Dans ce cas, l'exécution sera souvent à reprendre au début (cf. plus haut, le caractère d'indivisibilité).

- Disponibilité. Les automatismes constituent des unités d'activité toute prêtes, immédiatement exploitables, sans recours à la conscience sauf parfois pour leur déclenchement. "Les chaînes opératoires machinales sont le fondement du comportement individuel ; elles représentent chez l'homme l'élément essentiel de la survie. Elles se substituent à l'instinct dans des conditions proprement humaines puisqu'elles représentent un niveau élevé de disponibilité cérébrale. On ne peut, en effet, imaginer ni un comportement opératoire qui exigerait une complète lucidité, ni un comportement totalement conditionné qui ne la ferait jamais intervenir ; l'un parce qu'il aboutirait à réinventer le moindre geste, l'autre parce qu'il correspondrait à un niveau complètement pré-conditionné et par conséquent inhumain » (Leroi-Gourhan, 1965, p. 29).

- Autonomisation. «L'acquisition d'automatismes est indissociable d'une stratégie d'autonomisation » (Lelong, 2002, p. 281). Cet auteur a confirmé cette affirmation par l'étude de particuliers qui s'initient à l'usage d'Internet. Il cite cette déclaration d'un internaute : « on arrive plus ou moins à se débrouiller quand on a appris par soi-même » (id.) et il conclut : « Les internautes s'efforcent de développer des compétences totalement intériorisées, activables sans recourir à une personne, un texte ou un objet » (id.).

- Support à l'acquisition de compétences supérieures. Les avantages des automatismes en font un support indispensable à l'acquisition de compétences de niveau élevé. C'est parce que des composantes de ces compétences ont été automatisées que ces compétences peuvent se développer. 
Par exemple, c'est parce que la lecture est devenue un comportement automatisé que l'utilisation d'instructions, de consignes écrites pourra répondre à leurs contraintes d'utilisation. On conçoit facilement les difficultés que peut rencontrer dans la vie courante, et plus encore dans la vie de travail, celui qui n'a pas automatisées les opérations simples de calcul de l'addition et de la multiplication. Comme l'avait pertinemment noté James (1890, cité par Schneider et Chein, 2003, p. 555), « plus nombreux seront les détails de notre vie quotidienne qui pourront être confiés à la garde d'automatismes économes d'effort, plus les capacités supérieures de notre esprit seront libérées pour leur propre travail ». C'est par la mise en œuvre et l'articulation des deux modes de fonctionnement cognitif - contrôlé et automatique - que ce dernier pourra trouver son maximum d'efficacité.

- On lira une belle illustration des analyses précédentes dans les réflexions d'un Maitre d'Art sur sa pratique de potier. "Seule la répétition du geste conduit à sa liberté ; s'il n'est pas intégré dans l'inconscient du corps, c'est la matière, l'outil, la force centrifuge ou la pesanteur qui auront le dessus. La plupart des tâches du potier exigent des gestes répétitifs qui, acquis, deviennent des automatismes. Le broyage, le tamisage, le pétrissage, le tournage, le tournasage, le mélange des produits pour les couvertes, le polissage du pied après cuisson sont des opérations de nature très physique qui se font sans le concourt de la réflexion, ni de la volonté. On pourrait les croire fastidieuses, ce sont au contraire des moments de grande liberté où, le corps occupé, l'esprit est libre de vagabonder, de méditer sur les pièces à venir, ou de tout simplement s'abandonner au moment présent ». (Girel, 2004, p. 84)

\section{2.- Éléments pour une bonne gestion des automatismes}

Les automatismes présentent des effets négatifs lorsqu'ils se figent et se coupent de l'activité. Une bonne gestion doit donc s'efforcer de garder aux automatismes une ouverture afin d'éviter qu'ils se referment sur eux-mêmes au lieu de devenir les composantes d'actions plus larges. Dans ce but sont proposables deux types de mesures qui peuvent être rangées sous les rubriques « ce qu'il faut faire » et « ce qu'il ne faut pas faire. » On mentionnera seulement quelques mesures à titre d'illustration.

- Les mesures négatives. Elles consistent à éviter les conditions favorables au renforcement des automatismes quand celui-ci est jugé excessif. On citera notamment : - Eviter la répétition prolongée de la même tâche dans des conditions à faible variabilité. - Eviter la parcellisation des tâches : plus la tâche est réduite et pauvre, plus vite elle s'automatise avec la répétition et se referme sur elle-même. Cet effet se renforce quand la tâche considérée ne s'inscrit pas, pour l'opérateur, dans une tâche plus globale. - Eviter les contraintes de temps trop fortes car celles-ci conduisent à une organisation de l'activité à l'économie, rendant l'exécution de plus en plus indépendante du contexte, par l'élimination de contrôles toujours coûteux en cours d'exécution.

- Les mesures positives. On peut en imaginer beaucoup en miroir des mesures négatives : ajoutons deux catégories. - Introduire des éléments de variabilité dans le travail pour éviter la fermeture de l'automatisme dans un champ étroit. Ce rôle positif de la variabilité a été souligné par Falzon et Teiger (1995) qui parlent de « fonction constructive » de la « répétition sous variation » : celleci s'avérerait alors comme un antidote de la sclérose des automatismes. Cette variabilité peut être réalisée par l'élargissement des tâches, surtout si cet élargissement s'accompagne d'une plus large autonomie de l'opérateur. Celle-ci devrait amener à faire participer l'opérateur à la conception et à la gestion de ces tâches et à lui confier éventuellement des fonctions annexes comme l'entretien et l'évaluation. On a pu suggérer aussi des rotations de postes afin que l'opérateur élargisse ses compétences en même temps qu'il échapperait à une automatisation excessive. Falzon et Teiger notent aussi qu'on a pu observer que « des sujets devant exécuter une tâche répétitive introduisent spontanément de la variation (auto-générée) ... » (p. 3). Le même phénomène a été signalé par Clot (2001, p. 39). - Entretenir la justification cognitive de l'action, c'est- 
à-dire les connaissances sous-jacentes à la conception et aux modalités d'exécution. Quand ces connaissances s'appauvrissent, l'opérateur est enclin à recourir à l'automatisme bien rodé, fut-ce à mauvais escient, plutôt que de rechercher une solution originale issue de connaissances plus approfondies de la tâche, mais qu'il faut élaborer avec difficulté. L'effet positif de la variabilité est ainsi annulé.

- Recherche du compromis acceptable. Gérer les automatismes, c'est trouver un compromis acceptable entre leurs avantages et leurs inconvénients. La surautomatisation constitue un facteur de sclérose et surtout un obstacle à tout élargissement. L'opérateur qui a automatisé son activité à une tâche répétitive est souvent peu enclin à accepter une modification de celle-ci qui lui fera perdre le bénéfice de cette automatisation, en particulier l'allègement de la charge qui lui est associé. Il faut donc découvrir les conditions qui rendront ces changements acceptables en tenant compte de la difficulté qu'ils représentent pour l'intéressé. Le problème majeur est ainsi de concevoir une organisation du travail dans laquelle l'opérateur puisse profiter de l'automatisation sans s'y enfermer dans un champ trop étroit. Ce compromis est difficile et met en jeu des facteurs organisationnels, économiques et sociaux : c'est dire que ce compromis ne peut être improvisé et requiert une gestion complexe qui associe une pluralité d'acteurs et de compétences.

\section{3.- Automatismes et formation}

On retrouve les questions précédentes en abordant les rapports entre automatismes et formation. On en retrouve aussi de plus spécifiques qui tournent autour de la question : quelle place faire aux automatismes dans une formation? Pendant longtemps, dans les formations professionnelles, un principe généralement accepté était qu'on apprend d'abord à bien faire avant de faire vite. Sous-jacent à ce principe, est l'hypothèse qu' un automatisme qui permet une exécution plus rapide consiste à faire la «même chose» plus rapidement. Or les analyses précédentes auront montré que cette vue est radicalement erronée : l'acquisition de l'automatisme se fait par la transformation de l'action initiale et alors, faire vite, c'est faire autrement (Leplat, 1970, in Leplat, 2002). Ce nouveau principe a pu conduire à faire de la contrainte de vitesse un moyen d'apprentissage en tant que cette contrainte amène précocement le sujet à organiser autrement son action. L'exemple des méthodes d'apprentissage à la lecture rapide illustre cette idée.

On en trouve une autre illustration dans des recherches consacrées aux apprentissages adaptatifs (pour un commentaire plus détaillé, cf. citation précédente). Le principe de ces apprentissages est de faire travailler le sujet à un même niveau de difficulté au cours de son apprentissage. Une variable de réglage souvent utilisée à cette fin est la vitesse : on règle les contraintes d'allure de façon que la difficulté (évaluée par des indices prélevés sur le comportement) soit constante. Pask (1965, cité par Leplat, 1970) avait donné un bon exemple de ce type d'apprentissage. Il s'agissait de faire apprendre la perforation de cartes mécanographiques à partir d'un clavier de dix touches. «Le calculateur peut présenter les signaux à une allure variable qui tient compte des résultats antérieurs du sujet. Si celuici répond correctement, la vitesse est accélérée, s'il fait des fautes, elle est diminuée. Il existe, comme le dit l'auteur, une relation partiellement compétitive et partiellement coopérative entre la machine et l'élève» (Pask, in Leplat, 1970, p. 109). L'apprentissage adaptatif permet de faire travailler constamment le sujet au niveau de difficulté jugé optimal, tenant compte du fait que quand la tâche est trop facile, l'ennui apparaît et l'attention se relâche ; quand elle est trop difficile, le sujet est découragé par l'échec et la motivation diminue. Ces expériences peuvent être considérées comme des exemples de guidage possible vers l'acquisition d'un automatisme, bien qu'elles ne semblent pas avoir été poursuivies jusqu'à ce stade, ni envisagées dans cette optique. Elles montrent bien que les contraintes de vitesse constituent un facteur d'évolution de l'organisation de l'activité, lequel reste toujours à manipuler correctement car il peut aussi contribuer à la désorganisation de l'action quand il est utilisé sans précaution. 
Une autre question importante pour la formation est, sans doute, l'identification des sous-tâches qui se rencontrent dans plusieurs tâches constituant le travail d'un agent. Ce sont celles-ci qui auront avantage à être automatisées et dont l'automatisation présentera le moins de danger puisqu'elles sont intégrées à des tâches variées. Un opérateur qui travaille dans la mécanique aura à automatiser l'usage d'instruments de mesure, de tableaux à double entrée, de codages divers, etc. La secrétaire aura à automatiser l'usage du clavier d'ordinateur, l'usage des logiciels courants, des formules de courrier, etc. Pour la détermination de ces éléments de base, on retrouve les problèmes évoqués pour la définition du contenu des enseignements polytechniques qui cherchent à dégager les opérations de base communes à plusieurs postes de travail. Il y aura toujours à tenir compte de l'articulation de ces automatismes de base avec l'action dans laquelle ils s'inscrivent.

Ces quelques aperçus n'épuisent pas les questions que pose à la formation la place des automatismes et le degré auquel leur acquisition doit être conduite : ils suggèrent que ces questions méritent d'être approfondies plus qu'elles le sont actuellement.

\section{4.- La modification des automatismes}

La création de nouvelles tâches ou la modification d'une tâche peut entraîner des conflits avec les automatismes existants. Olszweska (2002) a bien décrit les effets de la «rupture d'une action routinière ». Nous avons aussi évoqué plus haut les difficultés soulevées par la déstructuration de l'activité et l'importation de composantes automatisées d'une tâche dans une autre. Il faut aussi noter l'impossibilité de compter sur les seules instructions pour éliminer durablement ces sources de conflit. L'instruction peut être initialement observée par la mise en jeu d'une activité contrôlée, mais à mesure que l'apprentissage de la nouvelle activité progresse, des interférences surviennent, montrant que l'automatisme ancien n'est pas éteint. Ces interférences se produisent notamment quand le niveau d'apprentissage de l'activité nouvelle s'élève et tend lui aussi vers l'automatisme. Elles surviennent aussi dans les cas d'urgence ou lors de l'arrivée de contraintes inattendues.

La seule formation ne suffit pas non plus à compenser les inconvénients de dispositifs qui ne tiennent pas compte des automatismes acquis par l'opérateur. Commentant les inconvénients des couplages entre systèmes de signalisation et de commande, non conformes aux stéréotypes des opérateurs, nous écrivions (Leplat, 1972) : «Les inconvénients des mauvais couplages peuvent difficilement être éliminés par l'apprentissage. Certes, un apprentissage prolongé permet de réduire, voire d'annuler, la différence apparente d'efficacité des deux systèmes, mais l'introduction de contraintes dans la tâche (vitesse, tâches interférentes, par exemple) peut faire réapparaître l'automatisme correspondant au système initial. Garvey et Taylor (1959) qui ont établi expérimentalement ce point notent que «l'emploi de la formation pour optimiser la performance d'un système hommemachine peut, comme la tapisserie sur les fissures d'un mur, avoir des conséquences sérieuses et inévitables. Il est certain que si des systèmes doivent fonctionner de manière satisfaisante (...), la formation en l'absence de contraintes ne devrait pas être substituée à une conception satisfaisante de l'équipement » (p. 97).

Ces observations en rejoignent d'anciennes rapportées par Guillaume (1947) au chapitre traitant des interférences d'habitudes. Rappelant des études de psychologie expérimentale, cet auteur notait déjà qu' «il semble que deux habitudes opposées se supplantent successivement et qu'il ne reste pas de trace de la première quand la seconde a fini par s'établir solidement. Mais il n'en est pas toujours ainsi... (p. 120). Il ajoutait plus loin que «l'inversion d'habitude demande plus de temps que sa formation primitive » (...) et que «il ne faudrait pas croire que la contre-éducation a eu pour effet d'effacer purement et simplement la première. Changer d'habitude n'est pas désapprendre » ( $p$. 120). Ce problème peut être illustré par certaines situations de travail dans lesquelles l'opérateur étant amené à travailler sur des matériels différents peut être affronté à des couplages de systèmes inverses, par exemple, pousser un levier pour obtenir un effet qui est obtenu par tirer sur un autre, ou 
passer d'un système de codage à un autre. Guillaume note que par un apprentissage prolongé, on peut obtenir la réponse correcte au signal ambivalent, mais que « le passage facile de l'une à l'autre <vient> de leur conjugaison en une seule habitude supérieure » (p. 121).

On trouvera des modèles d'analyse et des exemples de modification d'automatismes chez Rabardel (1995) au titre des « processus d'instrumentation relatifs à l'émergence et à l'évolution des schèmes d'utilisation » (p. 137).

Pour réduire la prégnance de l'automatisme, voire pour éliminer ce dernier, on peut essayer de le faire expliciter par la verbalisation. On a pu parler à ce propos de «décompilation de routine » (Falzon \& Teiger, 1995). La prise de conscience de son action peut conduire l'opérateur à la modifier et à la reconstruire sur d'autres bases. C'est dans cette perspective que s'inscrit la démarche décrite par Clot (2004) pour essayer « de redonner une histoire aux activités 'arrêtées' en démontant le moule où elles s'étaient prises » (p. 16). Un moyen de le faire est de transformer la représentation que le sujet a de son activité en faisant juger celle-ci par un autre ou en lui présentant l'activité d'un autre répondant à la même tâche. Cet objectif peut être obtenu par la méthode d'autoconfrontation croisée.

\section{Conclusion}

Une réflexion sur les automatismes est toujours utile quand on s'intéresse à l'analyse de l'activité : la raison en est bien claire puisque les automatismes sont une composante souvent essentielle de cette activité. Mais, en général, l'intérêt porté à cette composante est beaucoup motivé par l'étude des erreurs, ce qui conduit à identifier automatisme et source d'erreur. Si cette identification s'est montrée particulièrement productive, elle ne devrait pourtant pas masquer le rôle positif des automatismes dans l'activité : c'est à quoi s'est attaché ce texte dont on résumera quelques enseignements en rappelant qu'il voulait essentiellement attirer l'attention sur un champ qu'il n'a qu'incomplètement exploré. Ce texte souffre d'être amputé de la prise en compte de ces aspects négatifs. Cette lacune aura des conséquences atténuées par le fait que ces dernières sont mieux connues.

- Les automatismes sont des composantes de l'activité qui relèvent de catégories diverses. Entre les actions contrôlées et les automatismes «purs », il existe des degrés caractérisés par la présence plus ou moins nette de certaines propriétés de ces derniers.

- Si l'accord est général pour reconnaître dans l'automatisme un élément important de l'activité, il reste à mieux connaître comment il s'intègre progressivement à cette activité en se transformant et en la transformant. Un automatisme vit à l'intérieur de l'activité avec laquelle il est dans une relation dialectique: son développement modifie l'activité en même temps qu'il est conditionné par elle. Aussi, l'analyse de la manière dont s'articulent les composantes contrôlées et automatisées de l'activité est-elle un élément essentiel de l'analyse de celle-ci.

- Sur le plan pratique de la gestion des activités, l'étude des automatismes est essentielle : comment se développent-ils, jusqu'à quel point ce développement doit-il être favorisé, comment ses effets négatifs peuvent-ils être prévenus? Le présent texte a essayé d'apporter quelques réponses et de suggérer quelques pistes pour les rechercher.

- Sur le plan théorique, on a vu que les automatismes constituaient une catégorie complexe dont l'analyse pouvait être abordée par plusieurs voies : nous en avons exploré quelquesunes, mais il en est beaucoup d'autres. Ces voies ne devraient pas être conçues comme concurrentes, mais comme révélant chacune des propriétés particulières des automatismes, plus ou moins importantes selon les situations considérées. 
- S'il ne fallait garder qu'une phrase de conclusion, ce serait qu'il faut veiller à ce que l'automatisme ne se ferme jamais sur lui-même, mais soit toujours un tremplin pour l'acquisition d'activités plus riches et plus larges.

\section{RÉFÉRENCEMENT}

Leplat, J. (2005). Les automatismes dans l'activité : pour une réhabilitation et un bon usage. @ ctivités, 2 (2), 43-68, http://www.activites.org/v2n2/leplat.pdf

\section{BIBLIOGRAPHIE}

Bedny, G.Z., \& Karwowski, W. (2004). Activity theory as a basis for the study of work. Ergonomics, 47 (2), 134-153.

Bourdieu, P. (1980). Le sens pratique. Paris: Les Editions de Minuit.

Camus, J.-F. (1988). La distinction entre les processus contrôlés et les processus automatiques chez Schneider et Shiffrin. In P. Perruchet (Ed.), Les automatismes cognitifs (pp. 55-80). Liège: Mardaga.

Cheng, P.W. (1985). Restructuring versus automaticity: alternative accounts of skill acquisition. Psychological Review, 12, 414-423.

Clot, Y. (1999). La fonction psychologique du travail. Paris: PUF.

Clot, Y. (2001). Psychopathologie du travail et clinique de l'activité. Éducation Permanente, 146, 35-50.

Clot, Y. (2004). Le travail entre fonctionnement et développement. Bulletin de Psychologie, 57 (1), 5-12.

Clot, Y., Faïta, D., Fernandez, G. \& Scheller, L. (2001). Entretiens en autoconfrontation croisée : une méthode en clinique de l'activité. Éducation Permanente, 146, 17-26.

Dusire, S. (2000). Naviguer dans un espace verbal : la construction de la conscience de la situation. Thèse de doctorat d'ergonomie. Paris: CNAM.

Falzon, P., \& Teiger, C. (1995). Construire l'activité. Performances humaines et techniques, $\mathrm{N}^{\circ}$ hors série, 3440.

Galpérine, P. (1966). Essai sur la formation par étapes des actions et des concepts. In A. Léontiev, A. Luria, \& A. Smirnov (Eds.), Recherches psychologiques en URSS (pp. 114-132). Moscou: Editions du Progrès.

Garvey, W.D., \& Taylor, F.V. (1959). The limitation of a «procrustean» approach to the optimization of manmachine systems. Ergonomics, 2 (2), 187-194.

Girel, J. (2004). La sagesse du potier. Paris: L'œil neuf éditions.

Guillaume, P. (1947). La formation des habitudes. Paris: PUF.

Hanson, N.R. (1958). Patterns of discovery. Cambridge: Cambridge University Press.

Houdé, O., Kayser, D., Koenig, O., Proust, J., \& Rastier, F. (1998). Vocabulaire de sciences cognitives. Paris: PUF.

Janet, P. (1973/1886). L'automatisme psychologique. Paris: Alcan, $4^{\circ}$ édition.

Jourdan, M., \& Theureau, J. (Eds) (2002). Charge mentale : notion floue et vrai problème. Toulouse: Octarès.

Lee, J.L., \& Anderson, J.R. (2001). Does learning a complex task have to be complex ?: A study in learning decomposition. Cognitive Psychology, 42, 267-316.

Lelong, B. (2002). Savoir-faire technique et lien social. In C. Chauviré, \& A. Ogien (Eds.), La régularité (pp. 267-292). Paris: Editions de l'Ecole des Hautes Etudes en Sciences Sociales.

Léontiev, A. (1972). Le développement du psychisme. Paris: Editions sociales.

Léontiev, A. (1975). Activité, conscience, personnalité. Moscou: Editions du Progrès.

Leplat, J. (1970). Vitesse et formation. In J. Leplat, Cl. Enard, \& A. Weill-Fassina (Eds.). La formation par l'apprentissage (pp. 74-112). Paris: PUF. [Texte reproduit dans Leplat, J. (2002). Psychologie de la formation (pp. 93 134). Toulouse: Octarès]. 
Leplat, J. (1972). La psychologie du travail en ergonomie. In M. Reuchlin (Ed.), Traité de pasychologie appliquée, tome 3 (pp. 61-136). Paris: PUF.

Leplat, J. (1985). Erreur humaine, fiabilité humaine dans le travail. Paris: A. Colin.

Leplat, J. (1988). Les habiletés cognitives dans le travail. In P. Perruchet (Ed.), Les automatismes cognitifs (pp. 139-172). Liège: Mardaga.

Leplat, J. (1997). A propos des compétences incorporées. In J. Leplat. Regards sur l'activité en situation de travail (pp. 140-156). Paris: PUF.

Leplat, J. (2002). Psychologie de la formation. Jalons et perspectives. Choix de textes (1955-2002). Toulouse: Octarès.

Leplat, J., \& Pailhous, J. (1981). L'acquisition des habiletés mentales : la place des techniques. Le Travail humain , 44 (2), 275-283.

Logan, G.D. (1988). Toward an instance theory of automatization. Psychological Review, 95 (4), $492-527$.

Leroi-Gourhan, A. (1965). Le geste et la parole. La mémoire et ses rythmes. Paris: Albin Michel.

Merleau-Ponty, M. (1942). La structure du comportement. Paris: PUF.

Merleau-Ponty, M. (1945). Phénoménologie de la perception. Paris: Gallimard.

Neves, D.M., \& Anderson, J.R. (1981). Knowledge compilation : mechanisms for the automatization of cognitive skills. In J.R. Anderson (Ed.), Cognitive skills and their acquisition (pp. 57-84). Hillsdale, New Jersey: Lawrence Erlbaum.

Newell, A., \& Rosenbloom, P.S. (1980). Mechanisms of skill acquisition and the law of practice. In J.R. Anderson (Ed.), Cognitive skills and their acquisition (pp. 1-56). Hillsdale, New Jersey: Lawrence Erlbaum.

Norman, D.A. (1981). Categorization of action slips. Psychological Review, 88, 1-15.

Norman, D. A. (1988). The psychology of everyday things. New York: Basic Books.

Norman, D. A. (1993). Things that make us smart. Reading, Massachusetts: Addison-Wesley.

Olszewska (2002). Réfléchir avec les yeux et les mains. In C. Chauviré, \& A. Ogien (Eds.), La régularité (pp. 235-266). Paris: Editions de l'Ecole des Hautes Etudes en Sciences Sociales.

Pascal, B. (1954). Euvres complètes, Paris: Gallimard, La Pléiade.

Perruchet, P. (Ed.) (1988). Les automatismes cognitifs. Liège: Mardaga.

Rabardel, P. (1995). Hommes et Technologies. Approche cognitive des instruments contemporains. Paris: A. Colin.

Rasmussen, J., Pejtersen, A.M., \& Goodstein, L.P. (1994). Cognitive systems engineering. New York: J. Wiley.

Ravaisson, F. (1997/1837). De l'habitude. Paris: Payot et Rivages.

Reason, J. (1993/1990). L'erreur humaine. Paris: PUF.

Reynaud, B. (1998). Les propriétés des routines : outils pragmatiques de décision et modes de cooordination collective. Sociologie du Travail, 4, 465-477.

Rognin, L., Salembier, P., \& Zouinar, M. (2000). Cooperation, reliability of socio-technical systems and allocation of function. International Journal of Human-Computer Studies, 52, 357-379.

Schank, R., \& Abelson, R. (1977). Scripts, plans, goals, and understanding. Hillsdale, New Jersey: Lawrence Erlbaum.

Schneider, W., \& Chein J.M. (2003). Controlled and automatic processing: behavior, theory, and biological mechanisms. Cognitive Science, 27, 525-559.

Schneider, W., \& Shiffrin, R.M. (1977). Controlled and automatic human information processing: I. Detection, search, and attention. Psychological Review, 84, 1-66.

Shiffrin, R.M., \& Dumais, S.T. (1980). The development of automatism. In J.R. Anderson (Ed.), Cogntive skills and their acquisition (pp. 111-140). Hillsdale, New Jersey: Lawrence Erlbaum. 
Shiffrin, R.M., \& Schneider, W. (1977). Controlled and automatic human information processing: II. Perceptual learning, automatic attending, and a general theory. Psychological Review, 84, 127-190.

Teiger, C., Laville, A., Desssors, D., \& Gadbois, C. (1997). Renseignements téléphoniques avec lecture de micro-fiches sous contrainte temporelle. Paris: Rapport du Laboratoire de Physiologie du Travail et d'Ergonomie, ${ }^{\circ} 53$.

Tricot, A., \& Chanquoy, L. (1996). La charge mentale. Psychologie Française, nº spécial, 41 (4), 313-318.

Varela, F.J. (1989). Autonomie et connaissance. Paris: Seuil.

Varela, F., Thompson, E., \& Rosch, E. (1993). L'inscription corporelle de l'esprit. Paris: Seuil.

Vergnaud, G. (1990). La théorie des champs conceptuels. Recherches en Didactique des Mathématiques, 10 (2-3), 133-170.

Wallon, H. (1982 / 1938). La vie mentale. Paris: Editions sociales.

Wallon, H. (1942). La conscience et la vie subconsciente. In G. Dumas (Ed.), Nouveau Traité de Psychologie (pp. 1-38). Paris: PUF.

Winograd, T., \& Flores, F. (1989). L'intelligence artificielle en question. Paris: PUF.

\section{RÉSUMÉ}

Les automatismes tiennent une grande place dans l'activité, mais sont souvent invoqués pour leurs conséquences négatives, notamment, pour le rôle qu'ils jouent dans la genèse des erreurs. Cet article, en réexaminant la nature des automatismes, montre qu'ils ont aussi des propriétés susceptibles de jouer un rôle positif dans l'activité. Pour procéder à cette réhabilitation, on rappellera tout d'abord les traits généraux des automatismes, puis on recherchera les caractéristiques et les conditions du développement de ces derniers. Une classification des automatismes sera proposée. Pour mieux situer la place des automatismes dans l'activité, on examinera la manière dont ils sont conçus dans différents modèles de l'activité communément exploités en ergonomie. Enfin, seront suggérées quelques voies pour une utilisation judicieuse des automatismes qui tire profit de leurs avantages et réduise leurs effets négatifs.

\section{MOTS-CLÉS}

Activité, attention, automatisme, charge de travail, couplage, formation, habitus, schème, routine.

\section{RESUMEN}

Los automatismos en la actividad: en pos de una reivindicación y de un buen uso de los mismos;

Los automatismos ocupan un lugar importante en la actividad pero, a menudo, sus consecuencias se hallan connotadas negativamente a causa del lugar que ocupan en la génesis de los errores. A partir de un examen crítico acerca de la naturaleza de los automatismos, este artículo muestra que también poseen propiedades susceptibles de jugar un papel positivo en la actividad. Para poder realizar esta puesta en valor, primeramente se examinarán los rasgos más generales que poseen los automatismos, para luego poder caracterizarlos y describir las condiciones que permiten su desarrollo, proponiendo a su vez una clasificación de los mismos. Asimismo, a fin de poder situar el lugar que ocupan dichos mecanismos en la actividad, se examinarán las formas en que son considerados en los diferentes modelos de la actividad, usualmente en ergonomía. Por último, se plantearán algunas pistas para la utilización razonable de los automatismos, de forma tal de poder sacar provecho de sus ventajas y reducir sus efectos negativos. 
PALABRAS ClAVE

Actividad, atención, automatismo, carga de trabajo, acoplamiento, formación, habitus, esquema, rutina. 